\title{
COMMENTARY: Characterization and targeting of Platelet-Derived growth factor receptor alpha (PDGFRA) in Inflammatory breast cancer
} (IBC)

Kenneth L. van Golen*

The laboratory for cytoskeletal physiology, Department of biological sciences, The university of delaware, The center for translational cancer research, The Helen F Graham Cancer Center, Newark, DE, USA

\section{Article Info}

\section{Article Notes}

Received: November 22, 2017

Accepted: December 04, 2017

\section{*Correspondence:}

Kenneth L. van Golen, Ph.D., 320 Wolf Hall, The University of Delaware Newark, DE 19716, USA;

Email: klvg@udel.edu

(c) 2017 van Golen KL. This article is distributed under the terms of the Creative Commons Attribution 4.0 International License.

\section{Keywords}

Inflammatory breast cancer

Platelet derived growth factor receptor alpha

\section{Inflammatory breast cancer (IBC)}

The term 'Inflammatory Breast Cancer' (IBC) was first introduced in 1924, when Drs. Lee and Tannenbaum used it to describe a phenotypically distinct and aggressive presentation of locally advanced breast cancer (LABC) ${ }^{1}$. Based on clinical and pathological findings IBC unified what was thought to be several distinct entities of LABC as a single disease. Clinically, Lee and Tannenbaum recognized what appeared to be a classical immune inflammatory response with erythema, edema, swelling, intense pain and peau d'orange appearance of the breast; thus, the use of the phrase "inflammatory" in the description of the disease. Pathological findings indicate the presence of tumor emboli invading the dermal lymphatic vessels of the skin overlying the breast. It is thought that the poor prognosis of this disease is due to its ability to rapidly disseminated via the dermal lymphatic system ${ }^{2}$. The lympho-angiogenic nature and tendency to invade dermal lymphatic vessels contribute significantly to the metastatic nature of this disease ${ }^{3}$.

Inflammatory breast cancer is arguably the most aggressive form of epithelial breast cancer. By the current Surveillance, Epidemiology and End Results (SEER) Program estimates, IBC accounts for $1-3 \%$ of breast cancers in the United States, yet IBC is responsible for $\sim 10 \%$ of breast cancer-related deaths ${ }^{4,5}$. Most IBC physicians, researchers and advocates estimate that the actual incidence of IBC is higher, potentially accounting for up to $10 \%$ of total breast cancers ${ }^{6}$. By definition IBC is a T4d tumor and patients are diagnosed with stage IIIB or IV disease. Most patients show axillary lymph node involvement and almost 30\% patients show gross distant metastasis in organs such as lungs, liver and bone at their first clinical presentation ${ }^{5,7}$. It is the metastatic behavior of IBC that accounts for the poor clinical outcome with a current 5- and 10year survival rates of less than $45 \%$ and 20 , respectively ${ }^{8}$.

The current standard therapy for treating IBC is aggressive and multidisciplinary, including systemic chemotherapy followed by radical mastectomy and finally radiotherapy. Adjuvant therapy such as hormone therapy for hormone receptor-positive tumors and antiERBB2 drug for ERBB2-positive tumors may also be used ${ }^{4}$. Overall this treatment is relatively effective, although as implied above, it is 
insufficient with long-term survival being significantly less than conventional breast cancer and results in severe side effects offering a poor life quality for the patients ${ }^{6}$.

\section{Targeting overexpressed platelet derived growth factor receptor alpha in IBC}

Our study demonstrates that the platelet derived growth factor receptor alpha (PDGFRA) is overexpressed and active in IBC and can be successfully targeted ${ }^{9}$. A subset of IBC patients demonstrated overexpression of an active form of PDGFRA, which corresponded to a significant decrease in metastasis-free survival rates (MFS). Further, we demonstrated that the receptor tyrosine kinase inhibitor, Crenolanib, significantly inhibited tumor growth rates in an orthotopic mouse model of IBC.

Overexpression and activation of PDGFRA is known to play a role in the progression of a number cancers such as gastrointestinal stromal tumors (GISTs) ${ }^{10}$. Typically, in these types of tumors, activation occurs due to accumulation of activating mutation(s). Using GIST patient samples harboring PDGFRA with activating mutations, we created a predictive model to interrogate a large number of IBC patient samples. Those IBC patients identified as "PDGFRA activated" showed a 52\% 5-year MFS (95CI, 0.360.76), whereas patients that were predicted "not PDGFRAactivated" showed better survival with 72\% 5-year MFS (95CI, 0.63-0.81; $\mathrm{p}=0.022$ ). MFS is a surrogate for overall survival rate, therefore demonstrating that the presence of an active PDGFRA is indicative of more rapid progression and a poor prognosis.

When we analyzed IBC cell lines, we demonstrated that they also harbored an active PDGFRA. Data that was not reported in our publication but was presented at the 2012 International Inflammatory Breast Cancer Consortium meeting, suggested that PDGFRA was not mutant in IBC cells, rather it is mislocalized to the cytoplasm of the cell due to a possible defect in glycosylation ${ }^{6}$. The IBC cells express all four PDGF ligands, however, PDGF-BB was expressed at the highest levels and was expressed at 5-fold higher levels than conventional breast cancer cells. Taken together our data suggests that PDGFRA is packaged with PDGF-BB but is not properly localized to the plasma membrane. Instead, the receptor appears to remain active and signal in the cytoplasm of the IBC cell.

Targeting of PDGFRA by receptor tyrosine kinase (RTK) inhibitors is standard therapy for several cancers including GISTs. Imatanib (a.k.a. Gleevec or STI571) is the standard therapy for many cancers and its action is to prevent activation of the RTK. Unfortunately, as these tumors harbor a constitutively active form of the RTK, they become Imatinib resistant. Crenolanib (CP-868-596) was developed to target active PDGFR. With regards to monolayer growth, we demonstrated that IBC cells were refractory to Imatinib treatment over a 4,000-fold dose range. In contrast, the IBC cell growth was significantly inhibited by Crenolanib treatment at doses as low as 0.05 $\mu \mathrm{M}$. A hallmark of IBC growth is the formation of tumor emboli in the dermal lymphatic vessels overlying the breast. Our laboratory developed an in vitro method to culture IBC cells as tumor emboli. In this system IBC cells were also refractory to Imatinib treatment but were highly sensitive to Crenolanib treatment, forming significantly fewer tumor emboli compared to vehicle control and Imatinib treated cells at all doses tested. Interestingly, Crenolanib did not lead to IBC cell death but instead cell cycle arrest in G2/M.

Crenolanib-induced cell cycle arrest led to a significant reduction in orthotopic IBC tumor growth. SUM149 IBC cells were injected into the mammary fat pad of 8-week old female athymic nude mice and grown to 200 $\mathrm{mm}^{3}$. Crenolanib treatment over a 10 day period led to a significant difference in vehicle versus treated tumor growth with tumors being $753.9 \pm 116 \mathrm{~mm}^{3}$ and $197.6 \pm$ $50.7 \mathrm{~mm}^{3}$ for control and treated tumors, respectively. Crenolanib treatment was halted and mice were allowed to progress, tumors doubled in size within a 5 day time period suggesting that the effects of the RTK inhibitor are tumoristatic.

Our study is the first to demonstrate that an active PDGFRA in IBC patients is associated with a shorter survival period. Further, our study suggests that PDGFRA can be effectively targeted with Crenolanib. The tumoristatic properties of Crenolanib, resulting in a G2/M arrest, might be exploited by combining the RTK inhibitor with chemotherapy.

G2/M arrest sensitizes cancer cells to radiation ${ }^{11,12}$ and also to chemotherapy ${ }^{13}$. Given the inferior results of the present aggressive treatment of IBC, new therapies are desperately needed and PDGFRA may provide a specific target that can be effectively exploited.

\section{References}

1. Lee BTN. Inflammatory carcinoma of the breast: a report of twentyeight cases from the breast clinic of Memorial Hospital. Surg Gynecol Obstet. 1924; 39: 580-595.

2. Kleer CG, van Golen KL, Merajver SD. Molecular biology of breast cancer metastasis. Inflammatory breast cancer: clinical syndrome and molecular determinants. Breast cancer research. 2000; BCR 2: 423-429.

3. VermeulenPB, van Golen KL, Dirix LY.Angiogenesis, lymphangiogenesis, growth pattern, and tumor emboli in inflammatory breast cancer: a review of the current knowledge. Cancer. 2010; 116: 2748-2754, doi:10.1002/cncr.25169.

4. Dawood S. Biology and management of inflammatory breast cancer. Expert review of anticancer therapy. 2010; 10: 209. doi:10.1586/ era.09.90.

5. Dawood S, Valero V. in Inlfammatory Breast Cancer: An Update (eds N. T. Ueno \& M. Cristofanilli) Ch. 3, 11-20 (Springer, 2012). 
6. van Golen, K. L. \& Cristofanilli, M. The Third International Inflammatory Breast Cancer Meeting. Breast cancer research : BCR 15, 318-321 (2013).

7. Dawood S Merajver SD, Viens P, et al. International expert panel on inflammatory breast cancer: consensus statement for standardized diagnosis and treatment. Annals of oncology : official journal of the European Society for Medical Oncology / ESMO. 2011; 22: 515-523, doi:10.1093/annonc/mdq345.

8. Woodward,WA Cristofanilli M. Inflammatory breast cancer. Semin Radiat Oncol. 2009; 19: 256-265. doi:S1053-4296(09)00045-9 [pii] 10.1016/j.semradonc.2009.05.008.

9. Joglekar-Javadekar M, Van Laere S2, Bourne M, et al. Characterization and Targeting of Platelet-Derived Growth Factor Receptor alpha (PDGFRA) in Inflammatory Breast Cancer (IBC). Neoplasia. 2017; 19: 564-573, doi:10.1016/j.neo.2017.03.002.
10. Corless CL Schroeder A, Griffith D, et al. PDGFRA Mutations in Gastrointestinal Stromal Tumors: Frequency, Spectrum and In Vitro Sensitivity to Imatinib. Journal of Clinical Oncology. 2005; 23: 53575364, doi:10.1200/jco.2005.14.068).

11. Liu X, Sun C, Jin X, et al. Genistein Enhances the Radiosensitivity of Breast Cancer Cells via G2/M Cell Cycle Arrest and Apoptosis. Molecules. 2013; 18, 13200).

12. Di Z, Sanyuan S, Hong L, et al. Enhanced Radiosensitivity and G2/M Arrest were Observed in Radioresistant Esophageal Cancer Cells by Knocking Down RPA Expression. Cell Biochemistry and Biophysics. 2014; 70: 887-891, doi:10.1007/s12013-014-9995-3.

13. Zhang X, Xu LS, Wang ZQ et al. ING4 induces G2/M cell cycle arrest and enhances the chemosensitivity to DNA-damage agents in HepG2 cells. FEBS Letters. 2004; 570: 7-12, doi:https://doi.org/10.1016/j. febslet.2004.06.010. 